\title{
Ueber Jodkalium (Jodnatrium), Jodalbacid und Jodipin.
}

\author{
Von \\ Professor Edvard Welander \\ in Stockholm.
}

Da über diese Mittel bereits eine Menge Aufsätze das Tageslicht gesehen haben, habe ich mich erst nach einem gewissen Zaudern dazu entschliessen können, die Anzahl derselben zu vermehren; die Ursache, weshalb ich dieses thue, ist die, dass es mir scheint, als seien allzu wenig Untersuchungen über die Absorption und Elimination des Jods bei der verschiedenartigen Einführung dieser Mittel in den Organismus ausgeführt worden, Untersuchungen, die doch ihre Bedeutung haben oder wenigstens haben können.

Aus der dermatologischen Klinik des Prof. Pi ck sind zwar von $\mathrm{F}$ i s c h el sehr sorgfältige Untersuchungen über die Absorption und Elimination des Jodipins veröffentlicht worden, deren Ergebnisse sehr gut mit den von mir erhalteneu übereinstimmen. Da ich indessen nicht nur über die Absorption und Elimination des Jodipins, sondern auch des Jodkaliums und des Jodalbacids Untersuchungen ausgeführt habe, will ich über meine Untersuchungen in Kürze berichten.

Das, was sowohl im Jodkalium (Jodnatrium), wie in den neuen Jodpräparaten Bedeutung hat, ist ganz sicher der Jodgehalt; Nobl sagt, und dieses sicher mit Recht, dass es nur der Jodcomponent in diesen Präparaten sei, der bei der Beurtheilung der therapeutischen Wirksamkeit derselben in die Wagschale fällt. 
Wie es nun das Jod vermag, namentlich bei der Syphilis diese kräftige therapeutische Wirkung auszuüben, wissen wir nicht. Möglich ist es ja, dass es unmittelbar auf die Bakterien oder die Toxine, von denen wir annehmen, dass sie die Ursache der syphilitisch pathologisch-anatomischen Veränderungen sind, zerstörend einwirkt, aber es kann ja auch möglich sein, dass es in einer uns unerklärlichen Weise auf diese bei der Syphilis auftretenden Zelleninfiltrate resorbirend einzuwirken vermag, ohne auf das Syphilisgift selbst einen directen Einfluss auszuïben.

Hiermit mag es sich nun verhalten wie es will - eines scheint für eine schnelle und kräftige Ausübung dieser Wirkung des Jods doch erforderlich zu sein, nämlich dass es schnell absorbirt wird, damit es überall im Organismus verbreitet werden kann; das therapeutische Resultat wird um so besser, je schneller und in je grösserer Menge das Jod in den Organismus kommt und in ihm remaniert.

Diese deutet ja die klinische Erfahrung an; wir wissen, dass wir wohl mit kleinen Dosen Jodkalium mitunter gute Ergebnisse erzielen, aber auch, dass wir mit diesen kleinen Dosen oft nicht im Stande sind, die fortschreitende Entwickelung schwererer syphilitischer Symptome, z. B. von Gehirnleiden, mehr oder weniger im Zerfall begriffenen gummösen Infiltrationen u. s. w., zu hemmen, was uns doch sehr oft, ja in der Regel, und dies schnell, mittels grossen Dosen Jodkalium gelingt. Wir wissen auch, dass eine beginnende günstige therapeutische Wirkung des Jodkaliums in der Regel bald aufhört, wenn wir aus dem einen oder anderen Grunde genöthigt gewesen sind, die Anwendung dieses Mittels abzubrechen.

Alles dieses findet seine ungesuchte Erklärung in der Absorption und Elimination des Jods - mag das Jod nun auf die syphilitischen Veränderungen im Organismus specifisch oder nicht specifisch einwirken. Ich will hier hinsichtlich der Absorption und Elimination des Jods nur auf einige meiner eigenen Untersuchungen hinweisen, über welche ich in einem Aufsatze in Nord. Med. Arkiv im Jahre 1874 berichtet habe. ${ }^{1}$ ) Von

1) Nâgra undersökningar om jodets upptagande i och afskiljande ur menniskokroppen. 
den Ergebnissen dieser Untersuchungen will ich hier Folgendes hervorheben.

Bei der Einführung von Jodkalium in den Organismus in Lösungen per os wird das Jod besonders schnell absorbirt, so dass es schon nach einigen Minuten im Harne nachzuweisen ist, bei der Eingabe von Jodkalium in Pillen aber, namentlich wenn dieselben alt und hart sind, weniger schnell. Bei der Einführung von Jodkalium in Lösungen per anum zeigt sich eine sehr schnelle und kräftige Absorption des Jods, kräftiger, gleich nachdem das Rectum durch einen Stuhlgang geleert worden, als wenn es mehr oder weniger mit Scyballa angefiullt ist. Bei der Application von Jodkaliumlösung auf intacter Haut und bei der Einreibung von Jodkaliumsalbe 15-20 Minuten täglich in dieselbe findet gar keine Absorption des Jods statt; bei der Application von Jodkaliumlösung auf wunden Hautflächen wird das Jod schnell absorbirt. Das Jod wird sehr schnell aus dem Organismus ausgeschieden; auch bei der Einführung sehr grosser Dosen Jodkalium kann Jod nicht länger als $\left.2-3-4^{\prime}\right)$ Tage darnach im Organismus nachgewiesen werden. Das Jod wird hauptsächlich durch die Nieren, aber auch im Speichel u. s. w. ausgeschieden.

Das Jod wird auch in der Milch ausgeschieden, doch bei verschiedenen Individuen in einem etwas verschiedenen Masse; durch die Milch wird das Jod in den Organismus des die Brust nehmenden Kindes übergeführt und dann in seinem Harn eliminirt, in welchem es in der Regel $1-2$ Tage länger als in

") In einem Falle von sehr schwerer Nephritis mit höchst bedeutender Anasarka nahm der Patient vom 26.|I. bis zum 6.|II. 3 Gramm Jodnatrium täglich. Eine starke Jodreaction konnte ich nachher bis zum 12.|II. nachweisen; den 13./II. war die Jodreaction ziemlich stark und den 14./II. schwach; den 15./Il. und später vermochte ich kein Jod nachzuweisen. Möglicherweise war hier die bedeutende Nephritis die Ursache, dass die Elimination des Jods langsamer als gewöhnlich geschah, doch ist es wahrscheinlicher, dass die Transsudation des Jods in die ödematös angeschwollenen Körpertheile und seine Vertheilung daselbst die Ursache bildete, weshalb es so lange dauerte, bis sich alles Jod aus dem Körper eliminirt zeigte, denn kurze Zeit vorher, wo der Patient dieselbe schwere Nephritis hatte, die Anasarca aber unbedeutender war, konnte ich im Harne nicht länger als drei Tage nach dem Aufhören mit der täglichen Einnahme von $3 \mathrm{Gr}$. Jodnatrium Jod nachweisen.

Arch. f. Dermat. u. Syph. Bd. LViI. 
dem Harn und der Milch der ihm die Brust gebenden Frau nachgewiesen werden kann, was seinen Grund natürlicherweise darin hat, dass die säugende Frau 1-2 Tage früher aufhört, Jod $\mathrm{zu}$ erhalten, als das Kind, welches ja in der jodhaltigen Milch noch 1-2 Tage Jod bekommt, nachdem die säugende Frau schon aufgehört hat, dieses Mittel anzuwenden. Das Jod geht mit der grössten Leichtigkeit auf die Frucht in utero über - bei allen Versuchen, die ich gemacht habe, während der Entbindung der Mutter per os 1/2 Gr. Jodkalium zu geben, war Jod gleich nach der Geburt im Harn des Kindes nachzuweisen. Diese Untersuchungen zeigen jedoch nicht, wo im Organismus sich das Jod findet, und auch nicht, in welcher Menge es bei einer Behandlung mit Jodkalium daselbst vorhanden ist. Um dieses zu ermitteln, führte ich im Jahre 1896 einige Intersuchungen aus, welche ich in einem Aufsatz in der Wiener klin. Rundschau im Jahre 1897 veröffentlicht habe. ")

Diese Untersuchungen zeigen, dass Jod nur in unbedeutender Menge und nur kurze Zeit im Blute und auch in anderen Körpersäften nachzuweisen ist. Wenn ich z. B. um 8 Uhr Vormittags 1 Gr. Jodkalium gab, konnte ich um 9 Uhr Vormittags im Blute nur eine Andeutung von Jod finden, und um $10 \mathrm{Uhr}$ Vormittags und später in ihm keine Spur von Jod mehr nachweisen, ungeachtet sich im Speichel und im Harn noch mehrere Stunden nach der Einnahme des Jods eine sehr starke Jodreaction zeigte. Gab ich $3 \mathrm{Gr}$. Jodkalium auf einmal um 8 Uhr Vormittags, so zeigte sich im Blute um 8 Uhr 10 Minuten Vormittags eine recht starke, um 11 und 12 Uhr Vormittags und 2 Uhr Nachmittags eine weniger starke und um 4 Uhr Nachmittags eine schwache Jodreaction, um 6 Uhr Nachmittags nur eine Andeutung einer solchen Reaction, um 8 Uhr Abends eine höchst unsichere Reaction und später keine Spur einer Reaction mehr. Wurde z. B. 1 Gr. Jodkalium 4 Mal im Laufe des Tages, d. h. jede vierte Stunde gegeben, so war im Blute an diesem Tage eine Andeutung von Jod zu finden, am folgenden Tage aber keine Spur einer Jodreaction mehr zu entdecken. und 32 .

!) Einige Untersuchungen über Sod und Quecksilber. Nr. 29, 30 
Da nun die Hydrocelenflüssigkeit, die seröse Ergiessung in den Gelenken und die seröse Flüssigkeit in Vesicatorienblasen auf der Haut u. s. w. nur Transsudate aus dem Blute sind, erklärt es sich ja leicht, dass ieh in diesen Flüssigkeiten Jod nur unter denselben Verhältnissen und zu derselben Zeit wie im Blute, also nur in geringer Menge und nur kurze Zeit nach der Einführung von Jodkalium in den Organismus, finden konnte. Ganz anders verhielt es sich natürlicherweise mit einigen der Secrete, denn hier hatte ein Drüsenapparat den Uebergang des Jods aus dem Blute vermittelt, wodurch auch aus einem so jodarmen Blute, dass das Jod in ihm nicht nachzuweisen war, Jod in hinreichender Menge in das Secret abgesondert werden konnte, um in ihm eine starke Jodreaction zu geben; dieses war auch der Grund, dass sich z. B. in den hier genannten Fällen eine starke Jodreaction in dem Harne und dem Speichel sowie in anderen Secreten, z. B. im Cervicalsecret, fand, ${ }^{1}$ ) ungeachtet im Blute kein Jod mehr nachzuweisen war.

Man kann ja gegen diese Untersuchungen des Blutes bemerken, dass die bei ihnen angewandte Reaction auf Jod nicht empfindlich genug war; ich ging nämlich in der Weise zu wege, dass ich den Patienten schröpfte, das dabei erhaltene Blut 1-2 Tage in einem kleinen Glase auf Eis stehen liess, darauf das klare, gelbe Blutserum nahm und es mit Stärke (Oblatenpapier) und rauchender Salpetersäure untersuchte; diese Reaction ist, woren ich mich überzeugt habe, nicht empfindlicher, als dass sich im Harne, wenn auf 30.000 Theile Harn 1 Theil Jod kommt, nur eine Andeutung von Jod zeigt (siehe meinen

1) Diese Untersuchungen zeigen, in welch verschiedenem Masse die verschiedenen Drüsen Jod absondern, so z. B. die Drüsen in den weiblichen Genitalien; Jod konnte hier in der Regel nicht in dem Secret der Urethra]drüsen, der Barth. Drüse und der Vagina nachgewiesen werden, aber constant im Secret der Cervicaldrüsen; ob auch die Uterindrüsen Jod absondern, weiss ich nicht, dass es aber die Cervicaldrüsen thun, konnte ich aus der Jodreaction erkennen, die ich im Secret dieser Drüsen bei schwangeren Frauen fand; in der letzten Zeit habe ich hierfür einen noch sicheren Beweis erhalten; bei einer Frau war vor einem Jahre der ganze Uterus exstirpirt, die Cervix aber gelassen worden; bei der Einnahme von Jodkalium zeigte sich nun in diesem Cervicalsecret eine deutliche Jodreaction. 
Aufsatz). Berechnet man 1. Gr. Jodkalium als in der Blutmasse und den übrigen Körpersäften vertheilt, so kann man ja nicht erwarten, dass sich mit dieser Reaction :Jod im Blute nachweisen lässt, wenn nur eine so geringe Dosis wie 1 Gramm gegeben worden ist.

Ich habe deshalb Untersuchungen darüber angestellt, ob sich nicht durch eine genaue chemische Analyse des Blutes Jod im Blute auch in Fällen nachweısen lässt, wo ich mit der von mir angewandten Jodreaction kein Jod in ihm zu finden vermag, und, wenn dieses der Fall ist, wie lange sich das Jod wirklich im Blute finden kann. Herr Apotheker Blomquis t hat die Freundlichkeit gehabt, sämmtliche hier angeführten chemischen Analysen auszuführen (siehe hierüber seine Mittheilung am Ende dieses Aufsatzes).

Fall I. E. L. bekam seit rem 29./XI. 2 Gr. Jodkalium Morgens und Abends. Den 4.|XII. um 8 Uhr Vormittags erhielt die Patientin das letzte Mal $2 \mathrm{Gr}$. Jodkalium. Um $12 \mathrm{Uhr}$ Mittags erhielt ich in ihrem Blute mit Stärke und rauchender Salpetersäure eine deutliche Jodreaction; in gleichzeitig genommenem Blute fand B lomqu ist bei genauer chemischer Analyse eine ziemlich starke Jodreaction (Blaufärbung). ${ }^{\text {) }}$ ) Den 5./XII, um 12 Uhr Mittags konnte ich im Blute kein Jod nachweisen; Bl o m qu is t erhielt eine äusserst schwache Reaction von Jod. Den 6., 7. und 8.|XII. konnte weder Blomquist, noch ich Jod im Blate nacbweisen.

Fall II. O. L. nahm seit dem 30.|I. 2 Gr. Jodkalium Morgens und Abends. Den 3.|XII. um 8 Uhr Vormittags wurde Jod zum letzten Mal genommen; um 10 Uhr 15 Minuten Vormittags wurde von mir im Blute eine deutliche, aber schwache Jodreaction erhalten, von Blomqu i is t eine starke Blaufärbung und eir Niederschlag gefunden. Den 4.|XII. um 12 Uhr Vormittags vermochte ich kein Jod mehr im Blute zu finden, und auch Blo mqui st konnte solehes kaum in ihm nachweisen. Den 5.|XII. und 6. XII. vermochte weder Bl o $\mathrm{mqu}$ i s $\mathrm{t}$, noch ich Jod im Blute rachzuweisen.

B l o mqu is t konnte $z$ war bei genauer Analyse Jod in etwas grösserer Menge und auch eine etwas längere Zeit als ich im Blute nachweisen; es stimmen aber die Ergebnisse dieser Untersuchungen in der Hauptsache völlig mit den Ergebnissen meiner Untersuchungen vom Jahre 1896 überein, wo ich fand, dass das Jod sehr schnell (1-2 Tage) nach dem Aufhören mit der Einnahme dieses Mittels aus dem Blute verschwunden ist oder sich nur noch in so minimaler Menge in ihm findet, dass es nicht länger nachgewiesen werden kann. Dass nun auch im Harn durch eine genane chemische Analyse Jod längere Zeit als mittelst meiner weniger

1) Ueber die Bedeutung und Stärke dieser Reactionen siehe Blom$q u$ is t's beigefügte Mittheilung. 
empfindlichen Probe nachzuweisen sein würde, war ja wahrscheinlich, und dieses bewahrheitete sich auch bei folgender Untersuchung.

Fall III. G.P. hatte seit dem 2.|XI. täglich 6 Gr. Jodkalium genommen; den 28.|XI, bekam der Patient am Morgen 2 Gr. von diesem Mittel, worauf er mit der Einnabme desselben aufhörte. Den 30.|XI. um 8 Uhr Vormittags fand ich eine schwache, aber deutliche Jodreaction im Harne Blomquist hingegen einen blauen Niederschlag; nm 8 Uhr A bends fand ich im Harn Spuren von Jod, Blomqui st einen starken blauen Niederschlag. Den 1.jXII. und die folgenden Tage konnte ich kein Jod im Harne, nachweisen; Blomquist dagegen fand in jhm den 1.|XII. um 8 Uhr Vormittags einen ziemlich starken Niederschlag und um $8 \mathrm{Uhr}$ Nachmittags eine deutliche, nach 3 Minuten in das Violette übergehende Reaction. Den 2./XII. fand Bl.omquist im Harn um 8 Uhr Vormittags eine deutliche, unmittelbar in das Violette übergehende Reaction, um $8 \mathrm{Uhr}$ Abends eine äusserst schwache violette Färbung. Den 3,|XII. und auch die folgenden Tage konnte Blom quist kein Jod in Harne nachweisen.

Aus allen diesen Untersuchungen geht also hervor, dass bei der Einführung von Jodkalium in den Organismus per os eine sehr schnelle Absorption von Jod stattindet; dasselbe kommt in das Blut und geht mit demselben in die anderen Körpersäfte über, wird aber durch einige Drüsenapparate, namentlich die Nieren, sehr schnell, ja so schnell eliminirt, dass es selbst bei der Einnahme ziemlich grosser Dosen schon nach einem Tage beinahe ganz aus dem Blute verschwunden ist und nach 28 Stunden nur durch eine sorgfältigte chemische Analyse noch in äusserst geringer Menge und nach 48 Stunden gar nicht mehr in ihm nachgewiesen werden' kann. Dieses gibt uns ja ungesucht die Erklärung, weshalb die therapeutische Wirkung des Jodkaliums (was uns auch die klinische Erfahrung gelehrt hat) so schnell eintritt, aber auch, wenn die Einführung dieses Mittels in den Organismus abgebrochen wird, so schnell aufhört. l)ass wir ein Mittel, welches so schnell eliminirt wird und bei dessen Anwendung also von einer accumulativen Wirkung nicht die Rede sein kann, in grossen Dosen geben müssen, damit es sich in nennenswerther Menge im Blute und den anderen Körpersäften finden und (ganz sicher) dadurch eine kräftige therapeutische Wirkung auf vorhandene pathologisch anatomische Veränderungen ausüben kann, ist ja klar; dieses erklärt es uns ungesucht, dass oft grosse Dosen Jodkalium schnell die Entwickelung syphilitischer Veränderungen 
hemmen, auf welche die kleinen Dosen nicht einzuwirken vermögen.

Suchen wir nun aus dem einen oder anderen Grunde für das Jodbalium (Jodnatrium) ein therapeutisches Ersatzmittel, so müssen wir ja von diesem fordern, dass es, wie das Jodkalium, schnell und kräftig absorbirt werden kann; sollte es ausserdem vor dem Jodkalium den Vortheil haben, dass es nicht so besonders schnell aus dem Organismus eliminirt wird, so würde der Werth eines solchen Mittels höchst bedeutend steigen.

Wie verhält es sich nun in dieser Hinsicht mit den beiden Jodpräparaten Jodalbacid und Jodipin, die man besonders als geeignete und prächtige Ersatzmittel für das Jodkalium hat hervorbeben wollen?

In der ersten Zeit, wo ich Jodalbacid anwandte, konnte ich gar kein Jod im Harn nachweisen, auch dann nicht, wenn von diesem Mittel so grosse Dosen wie $4 \mathrm{Gr}$. täglich gegeben wordeu waren. Da ich es als wichtig ansah, zu wissen, ob bei der Behandlung mit Jodalbacid Jod absorbirt wird, und, wenn dieses der Fall, wie lange es nach dem Absehluss der Behandlung dauert, bis es vollständig aus dem Organismus eliminirt ist, bat ich den Apotheker B o o mqui st, in einigen Fällen, wo ich Jodalbacid angewendet hatte, eine chemische Analyse des Harns auszuführen. Es zeigte sich da, dass bei der Einnahme dieses Mittels Jod ziemlich schnell absorbirt wird, aber auch, dass seine Elimination aus dem Organismus ziemlich schnell geschieht; $\mathrm{Bl} / \mathrm{mq} \mathrm{a}$ ist konnte nämlich Jod nicht länger als bis zum 3. oder 4. Tage nach dem Aufhören mit der Einnahme von Jodalbacid mit Sicherheit im Harn nachweisen.

Bei fortgesetzter Anwendung des Jodalbacids fand ich indessen, dass Jod auch mit der von mir angewandten Untersuchungsmethode im Harn nachzuweisen war, und dieses (insonderheit in der lezten Zeit) sogar ziemlich bald, nachdem der Patient angefangen hatte, dieses Mittel einzunehmen. So begann z. B. Fall IV.

Fall IV. L. den 29./IX. um 2 Uhr Nachmittags mit der Einnahme von $1 \mathrm{Gr}$. Jodalbacid 3mal täglich. Am Abend des 30./IX. konnten von mir Spuren von Jod im Harn nachgewiesen werden. Den $1 \mid \mathrm{X}$. war die Jodreaction im Harne ziemlich stark. Am Morgen des 2./X. wurde das letzte Gramm Jodalbacid eingenommen; am Mittag war eine deutliche und am Abend um $8 \mathrm{Uhr}$ eine schwache Reaction im Harn nachzuweisen. Den 3./X. zeigte sich im Harn keine Jodreaction.

So auch im folgenden. Fall $\mathrm{V}$., wo der Patient den 27./XII. mit der Einnahme von $1 \mathrm{Gr}$. Jodalbacid 4 mal täglich begann. Den 28.jXII. war 
die Reaction im Harn recht stark. Den 29./XII. war die Reaction noch stärker, blauviolett bis blau, und dasselbe war auch den 2.JI. der Fall. Den 3./I. wurde um $8 \mathrm{Uhr}$ Morgens das letete Gramm Jodalbacid genommen. Am Morgen des 4.|I. fand ich kaum eine Andeutung einer Jodreaction im Harn. Den 5.|I. konnte ich gar kein Jod im IIarn nachweisen.

Ich habe deshalb die (vielleicht unrichtige) Auffassung bekommen, dass die Zusammensetzung des Jodalbacids sich allmälig verändert hatte (ob bei seiner Bereitung, oder bei seiner Aufbewahrung, kann ich nicht entscheiden) und dass das Jodalbacid solchergestalt, anstatt, wie ich glaubte, als ich anfing dasselbe anzuwenden, unzersetzt durch den Organismus zu gehen, wirklich zersetzt und zum Theil unter der Form von Jodalkali abgeschieden wird.

Hiemit magessich nun verhalten wie es will, so hat es sich doch stets gezeigt, dass das Jodalbacid zwar schnell absorbirt, aber auch schnell eliminirt wird. Es fragt sich da: kanndas Jodalbacid in therapeutischer Hinsicht mit dem Jodkalium verglichen werden? Ganz sicher, nein! Dasselbe hat vor dem Jodkalium, was die Absorption und die Elimination betrifft, keinen Vorzug, leider aber besitzt es die Ungelegenheit, dass es sehr arm an Jod ist, wovon es nur 10 Procent enthält, während sich in dem Jodkalium $\mathbf{7 6 . 5}$ Procent finden. Selbst wenn man täglich 4 Gr. Jodalbacid gibt, werden da in den Organismus nur $40 \mathrm{Cgr}$. Jod eingeführt, was ja eine kleine Dosis ist.

Es liesse sich hier ja einwenden, dass man, um eine kräftigere Wirkung zu erhalten, grössere Dosen Jodalbacid geben könne, denn in diesem Falle dürfte ja die Absorption von Jod und mithin auch die therapeutische Wirkung grösser sein, aber theils hindert die Theuerheit des Mittels seine Anwendung in grösseren Dosen, theils scheint man berechtigt zu sein, sich zu fragen, ob die Absorption des Jods bei der Einnahme dieser grösseren Dosen wirklich grösser wird.

Nach Ge yer's Angabe scheint dieses nicht der Fall zu sein, denn derselbe sagt in seinem Aufsatz "Das Jodalbacid und seine Verwendbarkeit in der Syphilistherapie" in der dem Herrn Hofrath Dr. J, N e u mann gewidmeten Festschrift: $n$. . Grössere Quantitäten Jodalbacid scheinen indessen nicht in entsprechendem Masse eine Jodausscheidung durch den Urin zu bedingen. Bei einer einmaligen Dosis von $150^{\circ}$ Jodalbacid früh 
Morgens 9 Uhr war um $1 / 210$ wenig Jod, um $1 / 2$ am meisten nachzuweisen, und die Proben Abends 10 und $11 \mathrm{Uhr}$ waren negativ. Dagegen erwies sich der aufgefangene Stuhlgang desselben Abends stark schwärzlichbräunlich gefärbt, zeigte einen intensiven Jodgeruch, und bei Untersuchung mit Salpetersãure und Chloroform liess sich eine deutliche Rothfärbung erzeugen. Das aufgenommene Jodalbacid wurde offenbar nicht vollständig im Darm verarbeitet."

Ich habe einen ähnlichen Versuch ausgeführt, wo jedoch nur $10 \mathrm{Gr}$. 'Jodalbacid gegeben wurden. Fall VI. D. bekam den 18./I. am Abend Pulv. Glycyr. Comp. und erhielt einen ordentlichen Stublgang den 19/I. um $6 \mathrm{Uhr}$. Vormittags. Um $8 \mathrm{Uhr}$. Vormittags wurden 10 Oblatenkapseln Jodalbacid, jede $1 \mathrm{Gr}$. enthaltend, gegeben. Im Harn fand ich um $8 \mathrm{Uhr}$ Vormittags kein Jod, auch nicht um 9 und 10 Uhr Vormittags. Um $11 \mathrm{Uhr}$ Vormittags war die Reaction violett; der Patient wurde geschröpft. Um $12 \mathrm{Uhr}$ Mittags war die Reaction im Harn blauviolett, um 1 und $2 \mathrm{Uhr}$ Nachmittags blau, um $6 \mathrm{Uhr} 30$ Min. und um $8 \mathrm{Uhr}$ Nachmittags stark blan. Um 6 Uhr 30 Minuten Nachmittags hatte der Patient einen Stuhlgang, der von etwas loser Beschaffenheit, im übrigen aber von normalem Aussehen war und keinen Jodgeruch hatte; in einem Theil der Faeces, in warmem Wasser aufgeschlämmt, konnte ich kein Jod nachweisen. Den 29.|I um $8 \mathrm{Uhr}$ Vormittags fand ich die Reaction im Harn blauviolett, um 12 Uhr Mittags eine Andeutung, um 3 Uhr Nachmittags kaum eine Andeutung einer Reaction; der Patient wurde da geschröpft; um 8 Uhr Nachmittags konnte ich kein Jod im Harne nachweisen. Im Laufo des Tages hatten sich beim Patienten Schnupfen und gelinde Kopfschmerzen eingestellt; diese Symptome pflegen bei ihm nach der Einnahme von Jodkalium stets aufzutreten. Am 21./I. konnte ich im Harn des Patienten kein Jod nachweisen.

B lo mqu ist konnte den 19.|I. um 9 Uhr Vormittags, also eine Stunde nach der Einnahme des Jodalbacids, im Harn des Patienten einen ziemlich starken, blaufarbigen Niederschlag nachweisen. Um 11 Uhr Vormittags fand sich im Harn ein ziemlich starker, blaufarbiger Niederschlag, während in gleichzeitig genommenem Blute gar kein Jod nachzuweisen war. In den Faeces um 6 Uhr 30 Minuten Nachmittags ein beinahe schwarzblauer Niederschlag. Den 20./I. zeigte sich um 8 Uhr Vormittags ein starker, blauer Niederschlag im Harn, und um 3 Uhr Nachmittags wurde in demselben eine blauviolette Reaction erhalten, während in gleichzeitig genommenem Blute kein Jod nachgewiesen werden konnte. Den 21./I. war um $8 \mathrm{Uhr}$ Vormittags im Harn noch immer ein blauer Niederschlag nachweisbar.

Diese. Untersuchungen zeigten zwar eine vermehrte Absorption des Jods, doch war dieselbe, wie Geyer sagt, durchaus nicht der eingenommenen grossen Dosis Jod, die hier 10 Gramm betrug, entsprechend; die Absorption war nicht so gross, dass sich in einer der genommenen Blutproben Jod hätte nach- 
weisen lassen. Dieses deutet ja mit ziemlicher Sicherheit an, dass wir von diesen grossen Dosen einen wirklichen Nutzen in therapeutischer Hinsicht nicht erwarten können.

Der grösste Theil des Jodalbacids ist in dem hier erwähnten Fall offenbar mit den Faeces abgegangen; eine Zersetzung des Jodalbacids in den Faeces konnte ich nicht entdecken. Aus den Untersuchungen scheint hervorzugehen, dass in dem untersten Theile des Darmes eine eigentliche Absorption des Jodalbacids nicht stattgefunden hat; wir hätten ja dann im Harne einen grösseren Jodgehalt finden müssen. Dass gleichwohl in dem untersten Theile des Darmes, im Rectum, eine Absorption des Jodalbacids stattfindet, obschon dieses nur in geringem Masse geschieht, ergibt sich aus folgenden Untersuchungen.

Fall VII. L. L. bekam Lakritzenpuiver (Pulv. Glycyrrh. Comp.) den 18.|I. und hatte davon 4 Stublgänge an diesem und 1 am Morgen des nächsten Tages, des 19./I. Nach diesem Stublgange wurden in das Rectum 2 Gr. Jodalbacid, in $25 \mathrm{Gr}$. Wasser aufgeschlämmt, eingespritzt. Erst um $10 \mathrm{Uhr}$ Abends hatte die Patientin wieder einen Stuhlgang. Bei 6 Untersuchungen am 19./I. war es mir nicht möglich, im Harne Jod nachzuweisen, und ebenso wenig gelang mir dieses den 20.|I. Blom quist fand den 19.|I. um $11 \mathrm{Uhr}$ Vormittags im Harne und auch im Blute kein Jod; dagegen konnte er im Harn, der um $8 \mathrm{Uhr}$ Nachmittags genommen wurde, Jod nachweisen, eine blaue Färbung erbalten. Den 20. I. um 9 Uhr Vormittags zeigte sich im Harne eine geringe Schattirung ins Violette.

Fall VIII. A. P. bekam ebenfalls den 19.|I. um 8 Uhr Vormittags 2 Gr. Jodalbacid in das Rectum eingespritzt; die Patientin hatte keine Lakritzenpulver erhalten, aber einen Stuhlgang gleich vor $8 \mathrm{Uhr}$ Morgens gehabt; sie hatte noch einen Stuhlgang um 4 Uhr 15 Min. Nachmittags. Auch bei dieser Patientin gelang es mir bei meinen Untersuchungen den 19.|I. und den 20./I. nicht, Jod im Harne nachzuweisen. Blomquist fand im Harn den 19./I. um 11 Uhr Vormittage kein Jod, um 8 Uhr Nachmittags eine rothviolette Färbung und den 20./I. um 9 Uhr Vormittags kein Jod. Also fand in beiden Fällen eine Absorption von Jod statt, wennschon dieselbe nur äusserst schwach war. Das geht aus diesen Fällen bervor, dass man zu therapeutischen $Z$ wecken nicht mit Fug Jodalbacid per anum geben kann.

Ich habe also bei dem Jodalbacid, mag es nun in grossen oder in kleinen Dosen gegeben oder mag es in der einen, oder in der anderen Weise in den Organismus eingeführt sein, keine solchen Eigenschaften gefunden, dass ich es in therapeutischer Hinsicht dem Jodkalium 
gleichstellen, noch viel weniger, dass ichesihm vorziehen kann.

So kommen wir zu dem Jodipin, welches theils per os, theils durch subcutane Einspritzungen in den Organismns eingeführt wird.

Bei der Eingabe von Jodipin per os findet eine schnelle und kräftige Absorption des Jods statt; das Jodipin wird so zersetzt, dass es wenigstens zum Theil als Jodalkali im Harn ausgeschieden wird und es sich mit Leichtigkeit mittelst der von mir angewandten Reaction (Oblatpapier und rauchende Salpetersäure) in ihm nachweisen lässt. Ebenso geht es schnell und in so grosser Menge in das Blut über, dass es auch in ihm leicht nachzuweisen ist.

Dieses war es z. B. im folgenden Fall, in Fall IX. D, bekam den 15.|I. um $8 \mathrm{Uhr}$ Vormittags $10 \mathrm{Ccm}$. 25proc. Jodipin per os; im Harn war vor der Einnahme des Jodipins kein Jod nachzuweisen. Um $9 \mathrm{Uhr}$ Vormittags war die Reaction im Harn blauviolett, um $10 \mathrm{Uhr}$ Vormittags blau, um $11 \mathrm{Uhr}$ Vormittags und um $12 \mathrm{Uhr}$ Mittags beinahe blauschwarz; um 4 Uhr und um 8 Uhr Nachmittags war sie beinahe schwarz, ebenso den 16.|I. um 8 Uhr Vormittags; um 8 Uhr Nachmittags war sie recht stark. Den 17.|I. um 8 Uhr 30 Min. Vormittags war die Reaction violett, um $12 \mathrm{Uhr}$ Mittags schwach violett. Bei der chemischen Untersuchung fand Blomquist den 15.|I. um $10 \mathrm{Uhr}$ Vormittags im Harn einen starken blauen Niederschlag und im Blut um 11 Uhr Vormittags eine blaue Färbung.

Fall X. 0. bekam den 17.|X. um 1 Uhr 30 Min. Nachmittags $5 \mathrm{Ccm}$. 25proc. Jodipin und den $18 . / \mathrm{X}$. um 12 Uhr 30 Min. Nachmittags $10 \mathrm{Cem}$. solches Jodipin per os. Den 19./X. am Abend eine ziemlich starke Jodreaction im Harn, den 20.|X. um 9 Uhr Vormittags eine ziemlich starke und um $3 \mathrm{Uhr}$ Nachmittags eine deutliche Jodreaction, sowie um $9 \mathrm{Uhr}$ Nachmittags eine Andeutung einer Jodreaction im Harn; den 22./X. um $9 \mathrm{Uhr}$ Vormittags sowie die folgenden Tage kein Jod im Harn nachweisbar. Das Jod war gleichwohl nicht ganz aus dem Harn verschwunden, denn den 24.|X. um $9 \mathrm{Uhr}$ Vormittags konnte Blomquist in ihm Spuren von Jod (eine violette Färbung, die in einer Minute verschwand) und den 27./X. Vormittags minimale Spuren von Jod (eine schwache, beinahe augenblicklich verschwindende rothe Färbung) nachweisen.

Jod hat also mehrere Tage nach seiner Einführung in den Organismus chemisch im Harne nachgewiesen werden können, aber nur ein paar Tage in einer grösseren, nachher in einer so minimalen Menge, dass e ine therapeutische Wirkung von ihm nicht denkbar ist. Per os in den Organismus 
eingeführt, wird das Jodipin zwar schnell und kräftig absorbirt, aber nicht so schnell und kräftig, wie das Jodkalium, daher es in dieser Hinsicht keinen Vorzug vor ihm hat; daza besitzt es eine grosse Ungelegenheit, nämlich die, einen so widerlichen, weichlichen Geschmack zu haben, dass die Patienten nicht gern mit seiner Einnahme fortsetzen wollen. Dieser Ungelegenheit würde sich zwar dadurch abhelfen lassen, dass man das Jodipin per anum gibt. Ich habe dieses in ein paar Fällen gethan, und zwar mit folgendem Resultat.

Fall YI. L. L. bekam den 10./I. lösende Mittel, worauf sie an diesem Tage drei Stuhlgänge, dann aber keinen Stuhlgang mehr vor dem 11./I. um 9 Uhr Nachmittags hatte. Den 11./I. um 8 Uhr 30 Min. Vormittags wurden per anum $15 \mathrm{Ccm}$. 25proc. Jodipin eingespritzt. Der Harn wurde von $9 \mathrm{Jhr} 30 \mathrm{Min}$. Vormittags bis um $12 \mathrm{Uhr}$ Nachts 10mal untersucht; auch nicht ein einziges Mal konnte ich Jod in ihm nachweisen, und dieses vermochte ich auch nicht bei 5 Untersuchungen im Laufe des 21/L.; hiernach untersuchte ich den Harn mit negativem Ergebniss 1-2mal täglich bis zum 16./. Es lässt sich indessen hieraus nicht der Schluss ziehen, dass hier kein Jodipin absorbirt worden ist, denn es konnte dieses geschehen sein, ohne dass das absorbirte Jodipin eine Zersetzung erlitten hatte, wodurch es für mich nicht nachweisbar war; es ist ja möglich, dass es unzersetzt durch den Harn eliminirt wurde. Dass dieses wirklich der Fall ist, zeigte die von Blomquist ausgeführte chemische Untersuchung. Um 10 Ubr 30 Min. (also 2 Stunden nach der Einspritzung per anum) fand Blomqui st Jod (eine schwache rosenrothe Färbung) im Harn; um 2 Uhr 30 Min. Nachmittags zeigte sich in ihm eine rothviolette und um 6 Uhr 30 Min. und 10 Uhr 30 Min. Nachmittags eine blauviolette Färbung. Den 12.I. um $8 \mathrm{Uhr}$ Vormittags wurde eine blaue Färbung, ein schwacher Niederschlag, erhalten. Den 13./I. um 9 Uhr Vormittags eine blauviolette Färbung und den 14./I. eine rosenrothe Färbung. Den 15.|I. und den 16./I. kein Jod. Also fand wirklich eine Absorption von Jod, obschon in sehr geringer Menge, statt; wir finden auch, dass das Jod sebr schnell eliminirt wurde.

In dem anderen Fall, Fall XII, wurden keine lösenden Mittel gegeben; der Patient, A. P., hatte eine natürliche Oeffnung den 11/.I. um $7 \mathrm{Ohr}$ Vormittags und am Abend; um 8 Uhr Vormittags den 11./I. wurden auch hier $15 \mathrm{Ccm}$. 25proc. Jodipin per anum eingespritzt; ich konnte weder an diesem noch an den folgenden Tagen Jod im Harn nachweisen. Blomquist fand in ihm den 12./I. um 12 Uhr 15 Min. kein Jod, den 13./I. um 9 Uhr Vormittags nur eine unbedeutende blauviolette Färbung, den 14./I. um 9 Uhr Vormittags eine schwache rosenrothe Färbung. Den 15./I. und die folgenden Tage fand er kein Jod. In diesem Falle fand sich also eine höchst unbedeutende Jodabsorption. Möglicherweise kann dieses darin seinen Grund gehabt haben, dass diese Person vor der Einspritzung des 
Jodipins keine Abführungsmittel orhalten hat und das Rectum daher nicht frei von Scybala gewesen ist. Da dieses bei der Einspritzung von Jodkalilösung per anum Einfluss auf die Absorption haben kann, ist es ja möglich, dass es auch bei der Einspritzung von Jodipin Einfluss auf die Absorption auszuüben vermag. Möglich ist es aber auch, dass sich eine individuelle Verschiedenheit vorgefunden hat; L. war nämlich eine junge, P. eine ältere Frau.

Wie es sich nun aber hiermit auch verhalten mag, so war doch die Absorption von Jodipin in beiden Fällen äusserst gering, und dieses scheint zu zeigen, dass eine Einspritzung von.Jodipin per anum zu therapeutischen Zwecken keine Anwendung finden kann.

Man hat als das grösste Verdienst des Jodipins herrorgehoben, dass es subcutan eingespritzt werden kann, wodurch es möglich ist, in bequemer Weise grosse Dosen Jod einzugeben, sowie dass es sehr langsam eliminirt wird und es solchergestalt wochenlang im Körper zu remaniren vermag. Von den Untersuchungen, die ich über die Absorption und Elimination des Jods bei subcutanen Einspritzungen von 25proc. Jodipin ausgeführt habe, mögen hier folgende angeführt werden:

Fall XIII. L. H. bekam den 26./XI. nm 9 Uhr Vormittags $5 \mathrm{Gr}$. 25proc. Jodipin subcutan eingespritzt. Im Laufe dieses Tages wurde der Harn der Patientin 7mal und die folgenden Tage bis zum 4./XII. 2mal täglich untersucht, ohne dass ich eine Spur von Jod in ibm nachzuweisen vermochte; ebensowenig vermochte ich Jod im Blut, welches den 3./XII. um 12 Uhr 30 Min. Mittags genommen worden war, nachzuweisen. Gleichzeitig wurden von B lom quis t chemische Analysen des Harnes ausgeführt. Weder den 27, noch den 28 . oder 29. konnte er in ihm Jod entdecken. Erst den 30./XI. um 9 Uhr Vormittags findet sich aufgezeichnet: Spuren von Jod, Blaufärbung, in 2 Minuten ins Violette übergehend. Den 1./XII. zeigte sich eine Blaufärbung, die in 2-3 Minuten ins Violette überging, und dasselbe war auch den 2./XII. der Fall. Den 3./XII. wurde eine schwache, bald verschwindende violette Färbung erhalten. Im Blute war an diesem Tage kein Jod nachzuweisen. Es zeigte sich also, dass das Jod langsam und in so geringer Menge absorbirt wurde, dass es erst nach 4 Tagen chemisch nacbzuweisen war. (Wären die. Untersuchungen fortgesetzt worden, hätte sicher noch eine Zeit nach dem 3./XIL. Jod nachgewiesen werden können.)

In dem folgenden Falle, Fall XIV, E. K., wurde, den 3./XLI, ebenfalls eine Einspritzung von 5 Ccm. 25proc. Jodipin gemacht. Es gelang mir hier nicht, Jod im Harn oder im Blute nachzuweisen. B lo m qu i s t fand weder den 3./XII., noch den 4./XII. ím Harn oder im Blute Jod. Den 5./XII. und den 6./XII. zeigte sich im Harn eine blauviolette Färbung; in gleich- 
zeitig genommenem Blute war kein Jod nachzuweisen. Den 7./XII. fand sich im Harn eine ziemlich starke Blaufärbung, im Blute eine schwache violette Schattirung. Den 8./XII. zeigte sich dasselbe Verhältniss. Auch in diesem Falle geschah a]so die Absorption des Jods nicht schnell, obschon das Jod hier etwas schneller und kräftiger als im vorigen Fälle absor. birt wurde.

Diese Untersuchungen deuten ja an, dass das Jodipin, wenn auch nicht ganz und gar, so doch wenigstens zum grossen, wenn nicht zum grössten Theil eliminirt wird, ohne dass eine Bildung von Jodalkali stattfindet.

In den folgenden beiden Fällen wurde täglich eine Injection von $5 \mathrm{Ccm}$. 25proc. Jodipin gegeben.

Fall XV. A. Die erste Injection wurde den 23./XI. gegeben. Der Harn wurde von mir täglich 2mal untersucht; erst am Abend des 29./XI. fand ich eine Andeutung von einer. Jodreaction, worauf sich Jod täglich in etwas vermehrter Menge nachweisen liess, so dass sich den 8./XII., wo der Patient die letzte Einspritzung erhielt, eine ziemlich starke Reaction zeigte; in gleichzeitig genommenem Blute vermochte ich dagegen kein Jod nachzuweisen. Bei der chemischen Untersuchung fand Blomquist den 1./XII. im Harn einen starken blauen Niederschlag, ebenso den 8./XII, ; dagegen konnte auch er in dem an diesen Tagen genommenen Blute kein Jod nachweisen.

Fall XVI. E. bekam von dom 15./XI. bis zum 27./XI. täglich $5 \mathrm{Ccm}$. 25proc. Jodipin subcutan eingespritzt, worauf er wegen Influenza mit den Einspritzungen bis zum 30./XI. aufhörte, wo sie dann wieder aufgenommen wurden; er erhielt die letzte Einspritzung den 3./XII. Erst den 22./XI. konnte ich im Harn eine Andeutung von Jod nachweisen, worauf sich einige 'Tage eine sehr schwache Jodreaction zeigte, die dann zunahm und von dem 30./XI. an ziemlich stark war; im Blut, welches den 3./XII. genommen wurde, konnte ich kein Jod nachweisen. Blomquist fand im Harn den 26./XI. und den 3./XII. einen starken blauen Niederschlag, konnte aber im Elut, welches den letztgenannten Tag genommen wurde kein Jod nachweisen.

Auch in diesen Fällen konnte nach täglichen Jodipineinspritzungen mittelst der von mir angewandten Reaction Jod erst nach mehreren Tagen in minimaler Menge im Harn nachgewiesen werden; auch während der ganzen übrigen Zeit verblieb die Reaction sehr schwach, obschon sie sich bei Blomquist's Untersuchungen als ziemlich stark erwies, was ja auch zu zeigen scheint, dass der allergrösste Theil des Jodipins obne Zersetzung in Jodalkali eliminirt wird.

Ich babe einmat Gelegenheit gehabt, den Uebergang des Jods durch die Milch von der Mutter auf das ibre Brust nehmende Kind zu studiren. Fall XVII. E. A. bekam den $2 /$ II. und den 3./II. $5 \mathrm{Ccm}$. und vom 4./II. bis zum 10./II. täglich $10 \mathrm{Ccn}$. 25proc. Jodipin. Den 6./II. konnte ich 
weder in dem Harn oder der Milch der Mutter, noch in dem Harn des Kindes Jod nachweisen. Blomquist fand Jod in dem Harne (einen blauvioletten Niederschlag) und der Milch (eine schwache rosenrothe Färbung) der Mutter, aber nicht in dem Harne des Kindes. Den 11./II. wurde die Mutter geschröpft, und ich vermochte da weder in der Milch und dem Harne, noch in dem Blute derselben Jod nachzuweisen, und ebenso wenig fand ich Jod im Harne des Kindes vom 10//II. B Io m q u i t fand in dem Blute der Mutter kein Jod, wohl aber in ihrer Milch (einen blauen Niederschlag) und in ibrem Harne (einen starken, blauen Niederschlag). Auch im Harne des Kindes fand sich eine starke Jodreaction, ein starker blauer Niederschlag. Im Harne der Mutter erhielt ich den 12./II. eine Jodreaction (rothviolett), in der Milch derselben aber vermochte ich kein Jod nachzuweisen.

Es geht also aus diesem Falle deutlich hervor, dass bei Einspritzungen von Jodipin Jod in nicht geringer Menge durch die Milch von der die Brust reichenden Frau auf das saugende Kind übergeht, und zwar, wie aus diesem einzigen Falle zu erhellen scheint, unzersetzt.

Als ein Beweis dafür, dass Jod bei den Jodipineinspritzungen lange im Körper remanirt, mag folgender Fall angeführt werden.

Fall XVIII. A. J. (Frau) bekam Injectionen von 5 Cem. 25proc. Jodipin vom 27.|X. bis zum 9.|XI., also 14 Tage. Der Harn wurde auf Jod, jeden Morgen und jeden Abend untersucht. Vom 3.|XI. bis zum 17./XI, wo die Patientin aus dem Krankenhause entlassen wurde, konnte ich beinahe bei jeder Untersuchung Jod im Harne nachweisen, obschon nur in so geringer Menge, dass sich in der Regel nur eine Andeutung einer Jodreaction gezeigt hat. Nach ibrer Entlassung aus dem Krankenhause untersuchte ich den Harn der Patientin drei Wochen zweimal wöchentlich mit demselben Ergebniss. Nach dieser Zeit konnte ich in ibrem Harn kein Jod mehr nachweisen. Blomquist konnte jedoch den 12.|XII., den 15./XII. und den 22./XII. einen starken blauen Niederschlag in ihrem Harn erbalten. Den 5.|I. erhielt er in demselben eine Blaufärbung, und den 19.|I. einen starken blauen Niederschlag. Ebenso den 2.|II.; 16./II. einen ziemlich starken blauen Niederschlag.

Also konnte Jod chemisch länger als drei Monate nach dem Aufhören mit den Jodipininjectionen im Harn nachgewiesen werden; je 99 Tage nach der lletzten Injection konnte Jod bei der chemischen Analyse in solcher Menge im Harn nachgewiesen werden; dass die Aufzeichnung lautet: "Ziemlich starker blauer Niederschlag." Dieses klingt so, als ob täglich eine grosse Menge Jod eliminirt worden wäre, was ja eigenthümlich erscheinen muss, da eine so geringe Anzahl Einspritzungen von nur je $5 \mathrm{Ccm}$. gegeben worden sind. Aber wenn anch das eliminirte Jod eine ziemlich starke Reaction gegeben hat, so ist damit durehaus 
nicht gesagt, dass seine Menge wirklich gross gewesen ist. Wie wir wissen, enthalten $5 \mathrm{Ccm}$. Jodipin beinahe $1.535 \mathrm{Gr}$. Jod und da die Patientin 14 Tage täglich $5 \mathrm{Ccm}$. Jodipin eingespritzt erhalten hatte, belief sich die in ihrem Organismus eingeführte Jodmenge anf ungefähr $21.5 \mathrm{Gramm}$. Noch den 16./II, also 113 Tage nach der ersten Einspritzung, konnte in dem Harn der Patientin Jod nachgewiesen werden. Wird nun die Elimination dieser 21.5 Gr. Jod auf diese 113 Tage vertheilt, so würde (vorausgesetzt, dass nach diesen 113 Tagen alles Jod aus dem Organismus eliminirt gewesen ist) täglich nur die geringe Menge von 19 Cgr. Jod eliminirt worden sein. Betrachten wir nun aber die von Blom quist angegebene Scala, so finden wir, dass die Reaction ${ }_{n}$ ziemlich starker blauer Niederchlag“ den 19./I. bei weitem nicht diese Menge eliminirtes Jod (25 Centigramm) angibt.

Approximativ berechnet zeigt diese Reactionsstärke, dass, ungeachtet die Reaction so stark erscheint, kaum ein einziges Centigramm Jod eliminirt worden ist. Bei einer so unbedeutenden täglichen Elimination kann es nicht Wunder nehmen, dass die in diesem Falle in den Organismus eingeführte Jodmenge, 21.5 Gramm, lange reichen konnte.

Es erschien mir eigenthümlich, dass es in den Fällen XVI, und XVII nicht einmal bei genauer chemischer Analyse möglich gewesen ist, Jod im Blute nachzuweisen. Um das Vorkommen des Jods im Blute bei diesen Einspritzungen näher zu erforschen, führte ich folgenden Versuch aus, bei welchem 10 Tage täglich $10 \mathrm{Ccm}$. 25proc. Jodipin eingespritzt und sowohl das Blut, wie der Harn alle Einspritzungstage und auch noch ein paarmal nach dem Aufhören mit den Einspritzungen untersucht wurden.

Fall XIX. B. erhielt die erste Einspritzung den 13.|XII., die letzte den 22.|XII. Erst den 21.|XII. vermochte ich in dem Harn des Patienten eine Jodreaction zu entdecken; ron diesem Tage ab habe ich aber bis zum 1.|I. beinahe täglich eine starke Reaction in ihm nachweisen können. Nach dieser Zeit hat sich noch bis zum 20.|I. täglich eine Jodreaction gefunden, die aber von wechselnder Stärke gewesen ist und sich einigemal recht stark, zumeist aber nur als eine Andeutung gezeigt hat. Im Blute konnte ich nicht ein einziges Mal Jed nachweisen. Blom qui st fand im Harn schon den 14.|XII., also am Tage nach der ersten Injection, eine schwache blaue Färbung, den 15.|XII. eine ziemlich starke blaue Färbung, den 16.|XIl. einen starken blauen Niederschlag und dann bis zum 23.|XII. täglich einen starken oder sehr starken blauen Niederschlag, also eine ziemlich kräftige Elimination. Trotzdem konnte er im Blute nur den 15.|XII., den 16.|XII. und den 17.|XII. eine äusserst schwache Jodreaction erhalten; die anderen Tage war es ihm nicht möglich, im Blute Jod nachzuweisen. Den 28.|XII. um $11 \mathrm{Ubr}$ Vormittags wurde eine neue chemische Unterebung angefürt, und bei derselben fand Blomquist im Harne 
Welander.

einen starken blauen Niederschlag und im Blüte eine schwache, aber deutliche rosenrothe Färbung. Den 15.|I. um 11 Uhr 15 Min. Vormittags wurde noch eine chemische Untersuchung ausgeführt. Bei derselben fand Blom quist im Harne noch immer einen starken blauen Niederschlag und im Blute eine Andentung von einer rosenrothen Färbung. Im Harne fand ich an diesem Tage eine schwache, aber deutliche Jodreaction. Ebenso den 16.|II.

Wir finden hier solchergestalt eine ziemlich schnelle $\mathrm{Ab}-$ sorption; Jod konnte hier, obschon nur in geringer Menge, bereits am Tage nach der ersten Einspritzung im Harne nachgewiesen werden. Die eliminirte Jodmenge wuchs täglich, so lange mit den Einspritzungen fortgesetzt wurde, und blieb viele Wochen nach dem Aufhören mit ihnen verhältnissmässig recht gross (starker blauer Niederschlag). Also fand sich auch hier eine lange andauernde Remanens von Jod im Organismus. Aber trotz alledem konnte Jod nur dreimal während der Einspritzungscur sowie bei den beiden Untersuchungen nach dem Abschluss der Behandlung und dazu nur in einer äusserst geringen Menge im Blute nachgewiesen werden.

Dieses muss ja die Frage hervorzwingen: wo im Organismus findet sich da das eingespritzte Jod während der Remanenszeit? Winternitz's an Thieren ausgeführte Versuche haben gezeigt, dass das in den Organismus eingeführte Jodipin in den subcutanem und anderem Fettgewebe sowie in den Muskeln u. s. w. abgelagert und von hier aus sehr allmälig wieder aus dem Organismus ausgeschieden wird.

Es erscheint mir als wahrscheinlich, dass das Jod, ehe es vollständig absorbirt wird, lange an der Einspritzungsstelle verbleibt. Andernfalls müsste man es wohl während der Zeit, wo die Einspritzungen gegeben werden, sowie in der nächsten Zeit darnach in grösserer Menge im Blute finden, denn durch dieses muss es wohl passiren, um in anderem Fettgewebe, in den Muskeln u. s. w. abgelagert werden zu können. Wenigstens spricht ein Fall, welchen zu untersuchen ich Gelegenheit hatte, dafür, dass es sich lange an der Einspritzungsstelle findet.

Fall XX. O. H. A. hatte vom 26.|X. bis zum 6.|XI. Jodipineinspritzungen von $5 \mathrm{Ccm}$. $(25 \%)$ bekommen; sie starb den 17.|XI. Bei der Obduction wurden für die Untersuchung auf Jod leider nur Fettheile von den Hinterbacken, wo die Einspritzungen gemacht worden waren, die Glandula thyreoidea, Blut und die Nieren aufbewahrt. Die eine Niere, 
in welche zwei Arterien führten, wurde so mit Wasser ausgespült, dass der eine Theil derselben kein Blut mehr enthielt und der andere (die nach diesem Theile führende Arterie war lädirt worden, damit nicht alles Blut ausgespült werden konnte) zum grössten Theil von seinem Blute befreit war. Die andere Niere wurde bei ihrem gewöhnlichen Blutgehalt untersucht. Es zeigte sich nun, dass in zwei Stückchen Fettgewebe von der Hinterbacke eine besonders starke Jodreaction (ein unbegrenżte Zeit bestehen bleibender blauer Niederschlag) erhalten wurde und auch in der Glandula thyreoidea eine sehr starke Jodreaction (ein sich mehrere Tage unverändert erhaltender blauer Niederschlag) nachzuweisen war. Im Blute, welches aus dem Herzen genommen war, liessen sich dagegen nur sehr schwache Spuren von Jod (ein schwacher Schimmer von einer blauvioletten Färbung) entdecken, und dasselbe war der Fall mit der Niere mit nicht vermindertem Blutgehalt; in dem theilweise von seinem Blute befreiten Nierenstückchen zeigte sich nur eine äusserst schwache, augenblicklich wieder verschwindende Schattirung ins Rothviolette, und in dem vollständig von seinem Blute befreiten Nierenstückchen war nicht eine Spur von Jod nachzuweisen. Die minimale Jodreaction in den mehr oder weniger bluthaltigen Nierentheilen schrieb sich natürlicherweise von dem Blute derselben her, und alles deutete darauf hin, dass sich im Nierengewebe kein Jod abgelagert fand. Die kleine minimale Jodmenge stimmt ja vollkommen mit der bei meinen früheren Untersuchungen des Blutes erhaltenen überein. Dass die Glandula thyreoidea das Vermögen besitzt, das Jod zurückzuhalten, ist schon lange bekannt; die in ihr erhaltene kräftige Reaction war also zu erwarten gewesen. Die besonders kräftige Reaction im Fettgewebe, wo die Einspritzungen gemacht worden sind, scheint mir zu zeigen, dass das dort eingespritzte Jodipin noch nicht absorbirt war; leider war kein anderes Fettgewebe genommen worden, daher ein Vergleich $z$ wischen dem Jodgehalt im Fettgewebe von verschiedenen Stellen nicht angestellt werden konnte.

Ich habe zwar in einem anderen Falle das Fettgewebe verschiedener Körpertheile auf Jod untersucht, bei dem Kinde in Fall XVII, wo die Mutter 9 Jodipineinspritzungen erhalten hatte. Dieser Fall beweist nichts, denn vor allem ist es, wenn auch wahrscheinlich, doch nicht erwiesen, dass das Jodipin unzersetzt durch die Milch auf das Kind übergegangen ist und das Kind solchergestalt wirklich Jodipin bekommen hat, und ebenso ist es, wenn dieses der Fall gewesen, nicht erwiesen, dass das Jodipin unzersetzt absorbirt worden und in diesem Zustande in die verschiedenen Körpertheile des Kindes gekommen ist; hierfür könnte zwar der Umstand sprechen, dass ich (mittelst meiner Untersuchungsmethode) im Harne des Kindes kein Jod nachzuweisen vermochte, ungeachtet Blomquist in ihm eine 
starke Reaction erhielt. Uebrigens war dieses kleine Kind sehr krank (es hatte Erysipelas), auch hatte es nur einige Tage jodipinhaltige (?) Milch erhalten, daher sich bei ihm kaum etwas Jodipin in dem Fettgewebe hatte ablagern können; das Kind starb nämlich am Tage nach dem Abschluss der Behandlung der Mutter mit Jodipineinspritzungen.

Es zeigte sich indessen, dass Jod nicht im Blute des Kindes, im Thymus oder in den Nieren nachzuweisen war; in der Leber wurde dagegen eine äusserst schwache Andeutung einer Jodreaction erhalten.

Fettgewebe wurde zur Untersuchung von beiden Hinterbacken (das Kind war sehr gut genährt), vom Omentum, vom Mesenteriurn und hinter dem Peritoneum genommen. Nur im Mesenteriumfett war eine äusserst schwache Jodreaction nachweisbar; in dem Fett von den anderen Stellen fand sich nicht eine Spur einer Jodreaction.

Auch die Thyreoidea wurde auf Jod untersucht; - in derselben fand sich eine sehr starke Jodreaction (ein starker blauer Niederschlag).

Ich will aus diesem Falle weder in der einen, noch in der anderen Hinsicht Schlüsse zu ziehen suchen, habe aber geglaubt, es nicht unterlassen zu dürfen, ihn hier anzuführen.

Wie nun die Jodipineinspritzungen eine therapeutische Wirkung ausüben können, wissen wir nicht. Sollte diese Wirkung von dem im Blute circulirenden Jod ausgeübt werden (was wenigstens als sehr wahrscheinlich erscheint, wenn das Jod unter der Form von Jodkalium per os in den Organismus eingeführt wird), so würden wir weder eine schnelle, noch eine kräftige therapeutische Wirkung des Jodipins zu erwarten das Recht haben. Dass eine solche Wirkung auch nicht ausgeübt wird, ist eigentlich meine Auffassung, aber theils besitze ich keine grosse Erfahrung, theils habe ich kleine Dosen Jodipin angewandt, daher ich mich nicht als berechtigt ansehe, dieses Urtheil auszusprechen.

Aber wir wissen ja, dass auch sehr kleine Dosen Jodkalium mitunter eine gute therapeutische Wirkung ausüben können.

Als ich vor mehr als 30 Jahren Assistenzarzt am Krankenhause für venerische Patienten in Stockholm war, wurde in der Regel nur $1 \mathrm{Gr}$. Jodkalium tăglich, ja mitunter noch weniger gegeben. Trotzdem konnte 
man in einer Menge Fälle allmälig ein gutes therapentisches Ergebniss erhalten; aber so fanden sich auch viele Fälle, in welchen diese kleine Dosis keine Wirkung ausübte. Von Jodalbacid, von welchem Mittel von mir in der R:gel so kleine Dosen wie 40 Centigr. Jod täglich gegeben worden sind, babe ich jedoch gute Ergebnisse gesehen (ich hàbe es aber nie gewagt, dieses Mittel in Fällen anzuwenden, wo eine schnelle Hilfe erforderlich war, wo eine schnelle und sehr kräftige Absorption sich als nothwendig erwies).

Wir wissen ja, dass in diesen Fällen, wo so kleine Dosen Jodkalium, wo nur $4 \mathrm{Gr}$. Jodalbacid gegeben worden sind, der Jodgehalt im Blute sehr klein gewesen ist, aber gleichwohl therapeutische Ergebnisse haben gewonnen werden können. Es scheint mir deshalb kein Wunder zu sein, dass diese Jodipineinspritzungen eine therapeutische Wirkung ausüben können, obschon bei ihnen nur eine minimale Menge Jod im Blute nachzuweisen ist. Aber eine andere Frage, die zu beantworten noch erübrigt, ist die, ob sie z. B. bei Gummata im weichen Gaumen, welche zu zerfallen drohen, oder in anderen Fällen, wo eine besonders schnelle und kräftige Jodwirkung von Nöthen ist, hinreichend kräftig wirken können.

Lin Verdienst der Jodipineinspritzungen ist ja die lange Remanens des Jods im Organismus, die es denkbar macht, dass das Jod dazu beitragen kann, dem Auftreten neuer tertiärer Symptome vorzubeugen.

Ich kann es nicht unterlassen, hier die Vermuthung auszusprechen, dass diese Jodipineinspritzungen, in einem frühen Stadium der Krankheit repetirt, möglicherweise für die Zukunft von grossem Nutzen sein können. Wir sind ja der Ansicht, dass die Tabes dorsalis (gleichwie die Mischungsform von Tabes und Paralysie générale) in der Einwirkung von Syphilistoxinen ihren Grund hat. Es fragt sich dann, wann diese Toxine gebildet werden. Wahrscheinlich ist wohl, dass ihre Bildung in die ersten (2-3) Jahre der Syphilis fällt, denn wir finden da Symptome, von denen wir annehmen müssen, dass sie durch Syphilisbakterien hervorgerufen sind. Oft sehen wir ja nach diesen ersten Jahren nicht ein einziges Symptom der Krankheit, bis die tabetischen Symptome anfangen, sich zu zeigen. Da sich nun das Jod als ein geeignetes Mittel gegen diese Symptome erwiesen hat, die wir auch in einem frühen Stadium 
der Krankheit als durch diese Syphilistoxine verursacht auffassen (z. B. schwere nächtliche Kopfschmerzen), so ist es doch wohl wenigstens möglich, dass diese gute Wirkung des Jods in einer Vernichtung der gebildeten Toxine besteht. Ist dieses nun der Fall, so lässt es sich ja denken, dass man durch eine beständige Eiuwirkung des Jods auf den Organismus während der Zeit, wo sich aller Grund findet zu argwöhnen, dass Syphilisbakterien noch vorhanden sind und somit Toxine fortfahrend gebildet werden, die Toxine in dem Masse, in welchem sie entstehen, möglicherweise zu vernichten und solchergestalt ihrer. künftigen Einwirkung auf das Nervensystem rorzubeugen und dadureh z. B. die Entwickelung von Tabes zu verhindern vermag. Mündlich bat mir Dr. Holmberg von Helsingfors mitgetheilt, dass er nicht nur denselben Gedanken gehabt, sondern auch praktisch in Uebereinstimmung damit gehandelt und seinen Patienten in den ersten beiden Jahren täglich kleine Dosen Jodkalium gegeben hat. Liegt nun wirklich etwas Gutes in einer solchen Einführung ron Jod in den Organismus, so würde dieselbe ja viel bequemer geschehen können, wenn periodenweise, z. B. jeden dritten oder vierten Monat, 10-15 Jodipineinspritzungen gemacht werden, als wenn eine so lange Zeit täglich Jodkalium gegeben wird; - in diesem Falle würde das Jodipin für uns von grossem Nutzen sein.

Wollten wir nun diese Mittel, was ihre therapeutische Kraft betrifft, mit einander vergleichen, so müssen wir wohl sagen, dass wir in keiner Weise im Stande sind mit Jodalbacid und Jodipin Jod im Organismus so schnell und in sogrosser Menge absorbirtzu erhalten und mit diesen Mittelnein so schnelles und so kräftiges therapeutisches Ergebniss zu erzielen, wie mit Jodkalium, mag dasselbe nun per os, oder mag es per anum in den Organismus gebracht werden. Wir können täglich 10 , ja $20 \mathrm{Gr}$. Jodkalium geben und solchergestalt dem Organismus täglich schnell 7, ja 15 Gr. Jod einverleiben. Das einzige Mittel, was in dieser Hinsicht in einer Weise mit dem Jodkalium verglichen werden kann, ist das. Jodipin per os eingegeben. Geben wir $10 \mathrm{Ccm}$. 25proc. Jodipin dreimal täglich, so können wir täglich $9 \mathrm{Gr}$. Jod in den 
Organismus einfuihren, es aber nicht so schnell absorbirt erhalten, wie bei der Eingabe von Jodkalium. In dieser Weise Jodipin zu geben, hat übrigens nicht den geringsten Vorzug vor der Eingabe derselben Menge Jod in der Form von Jodkalium - aber es ist damit die sehr grosse Ungelegenheit verbunden, dass die Eingabe ron Jod in dieser Weise sehr theuer sowie auch sehr unangenehm ist, denn das Jodipin hat einen sehr widerwärtigen, viel widerwärtigeren Geschmack als das Jodkalium.

Das Jodalbacid kann in Bezug auf die therapeutische Kraftnicht mit dem Jodkalium verglichen werden.

Da nun das Jodipin, subcutan gegeben, nicht besonders schnell absorbirt wird und nur in geringer Menge im Blute vorkommt, es aber lange im Organismus remanirt, scheint es mir mehr für die Anwendung in prophylaktischer, als in therapeutischer Hinsicht geeignet $z u$ sein; es ist zwar nicht erwiesen, doch liegt es innerhalb der Grenzen der Möglichkeit, dass das remanirende Jodipin vor Recidiven schützen kann.

(Das Jodipin und das Jodalbacid können - im Gegensatz zum Jodkalium - nicht mit Aussicht auf guten Erfolg in therapeutischer Hinsicht per anum gegeben werden.)

Nach einem Ersatzmittel für das Jodkalium zu forschen, würde wohl auch nicht in Frage gekommen sein, wenn nicht die Einführung von Jodkalium in den Organismus mit Ungelegenheiten verbunden wäre. Dieses ist leider mitunter der Fall, doch verträgt die grosse Mehrzahl der Patienten das Jodkalium recht gut, und für diese ist daher kein Ersatzmittel erforderlich; ausserdem haben wir in der Sulfanilsäure ein Mittel, welches, wenn nicht immer, so doch nicht selten diese Nebenwirkungen des Jodkaliums vermildert, ja sie sogar ganz aufhebt. Es scheint mir, als habe man in dem Bestreben, die Verdienste dieser Ersatzmittel hervorzuheben, all zu sehr und unberechtigt die Nachtheile der Anwendung des Jodkaliums betont.

So hat man hervorheben wollen, dass man bei schwereren Magenleiden und bei Gelegenheiten, wo die Patienten aus dem einen oder anderen Grunde das Jodkalium nicht hinunter- 
schlucken wollen oder können, so gut wie ausser Stande sei, Jod in den Organismus einzuführen, sofern man es nicht mittelst Einspritzungen von Jodipin thun will.

Man hat jedoch dabei allzu wenig beachtet, dass wir mittelst Einspritzungen von Jodkalilösung per anum Jod in einer viel schnelleren und kräftigeren Weise in den Organismus einführen können, als mittelst subcutanen Einspritzungen von Jodipin. In technischer Hinsicht dürfte man wohl in dẹr Regel auf keine grösseren Schwierigkeiten stossen, bei einer bewusstlosen Person, einem Irren u. s. w. Jodkalium per anum einzuspritzen, als hier, was nicht besonders schnell geht, subcutane Einspritzungen ron grossen Dosen Jodipin zu machen.

Schon 1874 habe ich hervorgehoben, dass bei diesen Einspritzungen ron Jodkalium per anum eine besonders schnelle und kräftige Absorption ron Jod stattfindet, und ich habe seitdem in einer grossen Anzahl von Fällen von dieser Anwendungsweise des Jodkaliums einen sehr grossen therapeutischen Nutzen gehabt. Ich spritze täglich $2-5$ mal 2 Gr. Jodkalium pro dosi in $25-30 \mathrm{Gr}$. Wasser ein, was in der Regel keine Reizung im Mastdarme hervorruft und auch nicht wie ein Klystier wirkt.

Ich will hier nur zwei der bei diesen Einspritzungen ausgeführten Untersuchungen über die Absorption des Jods anführen.

Fall XXI. L. L. bekam den 24.|I. Lakritzenpulver, worauf er einen Stuhlgang um 9 Uhr Nachmittags und einen um Mitternacht hatte. Den 25.|I. um 8 Uhr Vormittags wurden $2 \mathrm{Gr}$. Jodkalium per anum eingespritzt. Vor der Einspritzung war im Harn kein Jod nachzuweisen. Um 9 Uhr Vormittags fand ich die Reaction schwarz, ebenso bei 5 anderen Untersuchungen an diesem Tage, von denen die letzte um 8 Uhr Nachmittags ausgeführt wurde. Um 11 Uhr Vormittags wurde der Fatient geschröpft; im Blute fand Blomquist eine blauviolette Farbe. Den 21./I. um $8 \mathrm{Uhr}$ Vormittags war die Reaction stark blau, um 12 Uhr Mittags violett.

Fall XXII. A. P. haú kein Abführungsmittel bekummen. Den 21.|I. um $8 \mathrm{Uhr}$ Vormittags, wo sich in dem Harn der Patientin kein Jod fand, wurden per anum $2 \mathrm{Gr}$. Jodkalium eingespritzt. Um 9 Uhr Vormittags fand ich eine stark blaue, um 10 Uhr Vormittags eine schwarze Reaction im Harn; um 10 Uhr 15 Min. Vormittags batte die Patientin einen Stuhlgang. Um 11 Uhr Vormittags fand $\mathrm{Bl} O \mathrm{~m} q \mathrm{u}$ i st im Blute eine rothviolette Jodreaction. Im Harn fand ich die Reaction den 25.|I. schwarz; den 26. II. um 9 Uhr. Vormittags stark blau und um 1 Uhr Nachmittags violett.

Also eine äusserst schnelle und kräftige Absorption des Jods, welches in beiden Fällen auch im Blute nachzuweisen 
war. (Ich will hier auf den Unterschied aufmerksam machen, der sich bei diesen Versuchen, die bei denselben Frauen ausgeführt wurdeu, in der Absorption von Jod bei der Einspritzung von Jodkalium, von Jodalbacid und von Jodipin per anum findet.) Als ein Beispiel von dem Nutzen, welchen die Einspritzungen von Jodkalium per anum in therapeutischer Hinsicht machen, will ich hier nur folgenden Fall antühres.

Fall XXIII. G. wurde in halb bewusstlosem Zustande den 18.jVIII. 1900 in das Krankenhaus St. Göran aufgenommen und hatte bei seiner Aufnahme in das Krankenhaus unmotivirtes, intensives Erbrechen und Pupillen von verschiedener Grösse, war etwas paretisch in der rechten Körperhälfte und klagte über schwere Kopfschmerzen; er war frei von Fieber, was zusammen mit Narben von ulcerirten Gummata an den Beinen die Diagnose klar legte. Hier Jodkalium per os zu geben war des beständigen Erbrechens wegen nicht thunlich, daher der Patient 5mal täglich $2 \mathrm{Gr}$. Jorkalium per anum erhielt. Schon nach einigen Tagen hatte das Erbrechen aufgehört, der Patient war bei klarem Bewusstsein und sämmtliche Symptome zeigten sich verbessert (nachdem nun der Patient eine repetirte Behandlung mit Quecksilbersäckchen erhalten hat, ist sein Zustand äusserst befriedigend). Ich glaube nicht, dass ich dieselbe therapeutische Wirkung mit Jodipineinspritzungen erreicht haben würde, die ich hier, ohne Unbehagen für den Patienten, mit den Jodkaliumeinspritzungen per anum zu erzielen vermochte.

Schwerer sind dagegen die Einwendungen, die gegen die Nebenwirkungen des absorbirten Jodkaliums erhoben worden sind und unter denen wir eine bedeutende Menge Hautexantheme finden, die unangenehm zu sein vermögen, aber auch solche Leiden treffen, die nicht nur sehr unangenehm, sondern auch sebr gefährlich sein können, was namentlich von den Schleimhaut-Oedemen gilt, die sich, wenn sie sich im Schlunde und im Introitus laryngis localisiren, bei gesunden Personen als lebensgefährlich zu erweisen vermögen, dies natürlicherweise aber noch mebr bei Personen sind, bei denen schwerere syphilitische Veränderungen im Larynx Platz gegriffen haben. Hier kann es ja für den Patienten lebensgefährlich sein, Jodkalium zu geben, und es wäre daher wünschenswerth, ein Mittel zu haben, welches eine ebenso schnelle und kräftige therapeutische Wirkung wie das Jodkalium auszuïben vermag, von dem wir aber nicht solche Ungelegenheiten wie von dem Jodkalium zu befürchten haben. Es frägt sich dann, ob wir im Jodalbacid und 
im Jodipin wirklich solche Ersatzmittel für das Jodkalium gefunden haben.

Was nun die Ursache dieser unter vérschiedenen Formen auftretenden Nebenwirkungen des Jodkaliums ${ }^{1}$ ) anbetrifft, so kennen wir dieselbe wohl nicht sicher, doch ist es wahrscheinlich, dass das in das Blut gelangte Jodkalium zersetzt und dabei. Jod frei wird und dass hierin die eigentliche Ursache dieser unangenehmen Nebenwirkungen liegt. ${ }^{2}$ ) Ist diese Vermuthung richtig, so sollten wir ja diese Nebenwirkungen nicht bei Präparaten finden, die im Organismus nicht zu Jodalkali zersetzt werden, sondern ihn ganz und gar, oder doch wenigstens zum grössten Theil unzersetzt passiren.

Sehen wir nun nach, wie es sich hiermit beim Jodalbacid und beim Jodipin verhält, so will ich hier von den Einführungsweisen dieser Mittel in den Organismus nur diejenigen in Betracht ziehen, bei denen eine so grosse Menge Jod absorbirt wird, dass sich die Möglichkeit einer therapeutischen Einwirkung desselben denken lässt. Ich werde hier also nur die Einnahme des Jodalbacids und des Jodipins per os sowie die subcutane Einspritzung dieser Mittel näher betrachten.

Nun wissen wir, dass das Jodalbacid bei der Eingabe per os nur zum Theil zersetzt wird; da wir nun in der Regel nur verbältnissmässig kleine Dosen Jodalbacid (4 Gr. d. h. $0 \cdot 4 \mathrm{Gr}$. Jod täglich) geben und nur ein Theil dieses Jodalbacids als Jodalkali aus dem Organismus ausgeschieden wird, so ist es ja a priori wabrscheinlich, dass sich bei der Eingabe dieses Mittels in der Regel keine eigentlichen Nebenwirkungen des Jods zeigen. Im Anfange, wo ich mit der von mir angewandten Jodreaction kein Jod im Harne nachweisen konnte, was ja, wenn nicht zeigte, so doch andeutete, dass das Jodalbacid den Organismus unzersetzt (ohne in Jodalkali überzugehen) passirte,

1) Ich brauche hier nicht hervorzuheben, dass bei Herzaffectionen das Jodkalium gegen das Jodnatrium auszutauschen ist.

$\left.{ }^{2}\right)$ Für ihr Auftreten ist eine gewisse, bei verschiedenen Personen verschieden grosse Menge Jod im Blute erforderlich. Die Patienten können sich an das Jodkalium so gewöhnen, dass die Nebenwirkungen desselben narh einigen Tagen seiner Anwendung milder werden, ja sogar ganz aufhören. 
sah ich auch keine Nebenwirkungen des Jods; in der letzteren Zeit hingegen, wo ich stets Jod im Harne gefunden habe, habe ich mitunter auch Nebenwirkungen des Jodalbacids, obschon dieselben in der Regel ziemlich unbedeutend waren, gesehen.

Bei der Eingabe des Jodipins per os findet eine schnelle und kräftige Zersetzung desselben statt, so dass im Harn mit den gewöhnlichen Reagentien eine kräftige Jodreaction nachzuweisen ist. Wir haben da natülicherweise auch eine schnelle und kräftige Nebenwirkung des Jods zu erwarten; dass dieses der Fall ist, hat sich gezeigt.

Bei subcutaner Einspritzung des Jodipins sehen wir zwar, dass dasselbe als Jodalkali aus dem Organismus ausgeschieden wird, aber beinahe stets nur in geringer, ja sehr geringer Menge. Bei dieser Art der Einführung des Jodipins in den Organismus haben wir daher keine eigentlichen Nebenwirkungen des Jodipins zu erwarten; dieses hat auch die Erfahrung gelehrt.

Will man nun das Auftreten dieser Nebenwirkungen des Jods bei der Einführung der verschiedenen Jodpräparate in den Organismus studiren, so muss man dazu natürlicherweise solche Personen auswählen, bei denen diese Nebenwirkungen in einer mehr remarquabelen Weise auftreten, und ich will deshalb hier von allen den Fällen, die mir zu zeigen scheinen, dass die Nebenwirkungen des Jods ausbleiben, wenn das in den Organismus eingeführte Jodpräparat den Organisnuus beinahe gänzlich unzersetzt passirt, nur ein paar auführen.

Ich will jedoch erst zeigen, wie augenscheinlich der Unterschied in dieser Hinsicht zwischen dem innerlich gegebenen und dem subeutan eingespritzten Jodipin ist.

Fall XXIV. Bei einer Frau, bei welcher sich, nach der Einführung von Jodkalium in den Organismus stets Nebenwirkungen des Jods gezeigt hatten und bei welcher erst kürzlich solche aufgetreten waren, stellten sich bei der Behandlung mit Jodipineinspritzungen keine Nebenwirkungen des Jods ein; als aber die Fran während der Behandlung mit Jodipineinspritzungen einmal $10 \mathrm{C}$. C. Jodipin innerlich bekam, zeigten sich bei ihr sofort wieder die gewöhnlichen Nebenwirkungen des Jods, verschwanden aber, ungeachtet mit den täglichen Jodipineinspritzungen fortgefahren wurde, nach ein paar Tagen wieder.

Von den Fällen, wo sich eine ungewöhnlichere Nebenwirkung des Jods eingestellt hat, will ich hier folgenden anführen. 
Fall XXV, S erhielt den 25./X. um 7 Uhr Vormittags 2 Gr. Jodkalium wegen tertiärsyphilitischen Geschwüren. Nach drei Stunden stellte sich bei ihm ein unangenehmer Schnupfen ein, und nach 6-7 Stunden (im Laufe der Nacht) fing im rechten Augenlide ein Oedem an, sich zu entwickeln. Den 26./X fand sich um $12 \mathrm{Uhr}$ Mittags ein bedeutender rechtseitiger Schnupfen (die linke Nasenhälfte war völlig klar), ein bedeutender Thränenfluss aus dem rechten Auge und eine bedeutende Anschwellung im rechten Augenlide. Den 27./X. war das Augenlid weniger angeschwollen und der Schnupfen verschwunden. Am Abend wurden $5 \mathrm{Ccm}$. 100proc. Jodipin subcutan gegeben, was dann täglich bis zum 9.XI. geschah. Den 28./X. alle Anschwellung aus dem Augenlide versehwunden, keine Jodaffection - kein Jod im Laufe des Tages im Harne nachzuweisen. Den 29. $\mathrm{X}$. um $8 \mathrm{Uhr}$ Vormittags wurden per os $10 \mathrm{Ccm}$. 25proc. Jodipin gegeben; um 9, 10 und $11 \mathrm{Uhr}$ Vormittags starke Jodreaction im Harne; um $12 \mathrm{Uhr} 30 \mathrm{Min}$. Nachmittags kein Unbehagen vom Jodipin; wieder $10 \mathrm{Ccm}$. Jodipin per os. Um $3 \mathrm{Uhr}$ Nachmittags fing der Patient an sich unwohl zu füblen, und es stellte sich bei ihm jetzt linksseitiger Schnupfen ein, der sich mehr und mehr vermehrte; am Abend hatte er Kopfschmerzen, und während der Nacht fing das Athmen an ihm Schwierigkeiten zu bereiten; später des Nachts begann das linke Augenlied anzuschwellen, während sich im rechten keine Anschwellung zeigte. Den 30./X. um 7 Uhr Vormittags sowie auch um 12 Uhr Mittags sehr starke Jodreaction im Harn und das obere linke Augenlid ziemlich stark angeschwollen; der Patient ist etwas heiser und es findet sich ein gelindes Oedem im Introitus laryngis. Um $8 \mathrm{Uhr} 30 \mathrm{Min}$. Nachmittags starke Jodreaction. Den 31./X. um 8 Uhr Vormittags starke Jodreaction, noch Anschwellung im Augenlide, etwas heiser. Den 1./XI. um $8 \mathrm{Uhr}$ Vormittags schwache Jodreaction, das Oedema im Augenlide und die anderen Nebenwirkungen des Jods verschwunden. Der Patient fährt mit den täglichen Jodipineinspritzungen fort, wobei sich eine höchst unbedeutende Jodreaction im Harne nachweisen lässt; unter der Zeit keine Nebenwirkungen des Jods. Also Jodaffection nach der Einnahme von Jodkalium und Jodipin per os, aber nicht narb Jodipineinspritzungen.

In dem folgenden Falle gab das Auftreten einer ungewöbnlicheren Form von Joderythem zu erkennen, ob die Jodpräparate den Organismus beinahe unzersetzt passirten, oder ob sie in Jodkali übergingen.

Fall XXVI. B. begann den 18./II. 1900 mit täglicher Einnahme von 4 Gr. Jodkalium wider Sarkocele. Den 21./IL. hatte er an den Händen und Füssen sowie an den Knien einen dem Frythema multiforme ähnlichen Ausschlag in Ringen und Flecken bekommen; hörte da mit der Einnahme von Jodkalium auf. Den 23./II. war dieser Ausschlag verschwunden. Den 26./II. fing der Patient wieder mit der täglichen Einnabme von $4 \mathrm{Gr}$. Jodkalium an. Den 27./II. hatte sich bei ihm im Nacken, auf der Brust, auf dem Rücken und an den Schenkeln ein Erythema eingestellt. Den 28./II. hatte sich das Erythema an den genannten Stellen, Ringe and Flecken bildend, ausgebreitet. Den 1./III. hört der Patient wieder mit 
dem Jodkalium auf. Den 2./III. ist das Erythema bedeutend vermindert: den 6./III. ist das Erythema gänzlich verschwunden; der Patient fängt wieder mit der Einnahme von Jodkalium an. Den 8./III. bedeutendes Erythema am Körper; hört mit der Jodkaliumeinnahme auf. Den 10./VIII. das Erythema beinahe verschwunden. - Den 22./III. begann der Patient mit der Einnahme von $4 \mathrm{Gr}$. Jodalbacid täglich, mit welcher Einnahme er bis zum 26./III. fortfuhr. Den 27./III. kein Ausschlag aufgetreten; eine minimale Jodreaction hat sich im Harn gezeigt. - Den 28.|III. im Laufe des Tages dreimal $10 \mathrm{Ccm}$. Jodipin per os gegeben; schon zur Mittagszeit starke Jodreaction im Harn. Den 29./III. um 8 Uhr Vormittags starke Jodreaction; an den Händen und auf dem Rücken des Patienten zerstreute Erythemflecke, ein Theil derselben mit Vesiculae. Im Laufe des Tages dreimal $10 \mathrm{Ccm}$. Jodipin gegeben. Den 30./III. Morgens das letzte Mal 10 Ccm. Jodipin gegeben; sehr starke Jodreaction; der Ausschlag anf dem Rücken, auf der Brust und an den Händen ziemlich ausgebreitet und an mehreren Stellen kleine Vesiculae im Erythem. Den 1.|IV. noch kleinere Reste des Ausschlages vorhanden; keine Jodreaction im Harne. Den 2./IV. der Ausschlag ganz verschwunden. - Der Patient wurde im December von neuem in das Krankenhaus St. Göran aufgenommen und bekam da vom 13.|XII. bis zum 22.|XII. tägliche Einspritzungen von $10 \mathrm{Ccm}$. 25proc. Jodipin. Seit dem 21.|XII. war die Jodreaction im Harne ziemlich stark (blauviolett). Den 24./XII. zeigte sich das Erythema wieder, und zwar hauptsächlich auf dem Rücken, wo sich bis zum 4/I. welchselnde Erythemflecke und Erythemringe fanden; seitdem kein Erythem. Im Harne babe ich seit dem 4./I. stets eine Jodreaction gefunden, die aber im allgemeinen sehr schwach war, so auch den 21./I. - An diesem Tage bekam der Patient um 8 Uhr Vormittags 5 Gr. Jodalbacid auf einmal; um 11 Uhr Vormittags fand ich nur eine unbedeutende Jodreaction im Harne; um $2 \mathrm{Uhr} 30 \mathrm{Min}$. Nachmittags war die Jodreaction im Harne blau und um 8 Uhr 45 Min. Nachmittags violett. Den 23./I. um 11 Uhr 30 Min. Vormittags zeigte sich im Harne eine blauviolette und um $9 \mathrm{Uhr}$ Nachmittags eine schwach violette Jodreaction. Den 25/1. liess sich im Harne nur eine schwache Andeutung einer Jodreaction nachweisen. Dieses Mal blieb gleichwohl das Erythema aus, was seinen Grund wohl darin haben konnte, dass die Jodabsorption schwach war, und vielleicht auch darin, dass der Patient lange Zeit unter der Einwirkung von Jod gestanden batte; wir wissen ja, dass sich bei den Patienten im Anfange einer Behandlung mit Jod schwere Nebenwirkungen des Jods zeigen können, dass sie sich aber an dies Mittel gewöhnen und es dann ohne Ungelegenheiten zu vertragen vermögen. Wir sehen hier indessen dieses eigenthümliche Erythema bei der Einnahme von Jodkalium und Jodipin bald auftreten; bei den Jodipineinspritzungen zeigte sich nach 10 Einspritzungen zwar ein gelindes Erythema, doch verschwand dasselbe, ungeachtet sich noch Jod im Organismus fand, bald wieder. Das Jodalbacid rief kein Erythema hervor.

Ich babe in der letzten Zeit zwei Patienten gehabt, bei denen bei der Behandlung mit Jodkalium Blutungen in der Haut der Unterschenkel 
auftraten; dieselben verschwanden nach dem Abbruch der Behandlung wieder, doch traten sie bei beiden Patienten am Tage nach der Eingabe von $10 \mathrm{Ccm}$. 25proc. Jodipin von neuem auf. In beiden Fälen fand sich. natürlicherweise im Harne eine starke Jodreaction. - Schliesslich gab ich 4 Tage 4 Gr. Jodalbacid täglich, wobei keine Blutungen auftraten; die Jodreaction im Harn wurde hierbei blauviolett.

Sämmtliche diese Fälle legen dar, dass das Jodipin bei innerlicher Anwendung dieselben Nebenwirkungen des Jods wie das Jodkalium zeigt, bei Einspritzung des Jodipins sich aber keine oder nur unbedeutende Nebenwirkungen des Jods einstellen. Durch Anwendung dieser Einspritzungen würden sich also ziemlich sicher die Nebenwirkungen des Jods vermeiden lassen, und es würden uns diese Finspritzungen daher bei vielen Gelegenheiten, so z. B. bei tertiärsyphilitischen Veränderungen im Larynx, vom grossen Nutzen sein - wenn sich mit ihnen nur ein schnelles und kräftiges therapeutisches Ergebniss erreichen liesse. Die Untersuchungen, welche ich beiden Einspritzungen ron Jodipin über die Absorption des Jods a us eführt, und die Erfahrung, welcheich vondertherapeutischen Wirkung dieser Einspritzungengewonnen habe, lassen mich aber von ihnen keine grösseren Erwartungen in dieser Hinsicht hegen.

Die Rolle des Jodalbacids als Ersatzmittel für das Jodkalium scheint mir, da es nicht in grösseren Dosen gegeben werden kann und ausserdem bei seiner Anwendung mitunter dieselben Nebenwirkungen des Jods wie bei der Anwendung des Jodkaliums auftreten, keine grosse Bedeutung erhalten zu können.

Bis jetzt haben wir noch kein Mittel gefunden, welches die grosse therapeutische Kraft des Jodkaliums besitzt, aber frei von solche Nebenwirkungen wie dieses Mittel ist, daher das Jodkalium sicher noch immer den ersten Platz unter den Jodpräparaten einnimmt. Nur in Ausnahmefällen scheinen mir die Jodipineinspritzungen $\mathrm{zu}$ therapeutischen Zwecken anzuwenden zu sein - vielleicht werden sie eine Rolle in prophylaktischer Hinsicht spielen können. 


\section{Nachtrag.}

Ich habe Gelegenheit gehabt, auf Jod Leichentheile eines Foetus zu untersuchen, dessen Mutter gegen das Ende der Schwangerschaft Jodipineinspritzungen à $5 \mathrm{Ccm}$. vom 1 . bis zum 9. Feber erhalten hatte. Dieser Foetus hatte sich normal entwickelt. Den 6./III. wurden kräftige Herztöne des Foetus gehört und den 7./III. wurde die Mutter im Entbindungshause von einem ausgetragenen Kinde entbunden, welches während der ganzen Entbindung lebte, asphyktisch geboren wurde, nach einigen schwachen Versuchen zu athmen starb - ganz sicher in Folge des kurzen Nabelstranges - und nur 45 Centimeter lang war. An der Placenta sowie auch an dem Foetus waren keine syphilitischen Veränderungen nachzuweisen. In der Haut fanden sich zahlreiche kleine Ekchymosen sowie auch zahlreiche kleine subpleurale und subperitoneale Blutungen. Die Lungen waren zum grössten Theil ohne Luft, enthielten aber solche offenbar an einigen Stellen; sie schwammen zusammen mit dem Herzen in Wasser.

Von diesem Foetus wurden folgende Theile auf Jod untersucht:

Blut - 0 Jod, Lungen - 0 Jod, Nieren - 0 Jod, Thymus $-0 \mathrm{Jod}$;

Leber - Andeutung von Jod (schwache Schattirung);

Thyreoidea - bla uer Niederschlag;

Fett von der rechten Hinterbacke - augenblicklich verschwindende Rothfärbung;

Fett von der linken Hinterbacke - augenblicklich verschwindende Rothfärbung;

Retroperitonealfett - 0 Jod;

Mesenterialfett -0 Jod;

Placenta - 0 Jod.

Also war Jod (Jodipin?) durch die Placenta von der Mutter auf das Kind ïbergegangen und Jod fand sich 26 Tage, nachdem die Mutter die letzte Einspritzung erhalten hatte, in 
der Thyreoidea und in dem Fettgewebe der Hinterbacken abgelagert, ungeachtet nur eine so geringe Menge Jodipin eingespritzt worden war.

Im Falle XVIII konnte Jod (ein blauer Niederschlag) auch den 14./II. und den 2./III. nachgewiesen werden, und den 16./III., den 30./III., den 13./IV. und den 27./IV. fand sich auch eine starke Reaction (ein stark blau gefärbter Niederschlag). In diesem Falle liess sich also Jod 169 Tage nach der letzten Injection nachweisen. 